\title{
THE ORDER OF THE ANTIPODE OF A HOPF ALGEBRA
}

\author{
RICHARD GUSTAVUS LARSON ${ }^{1}$
}

The purpose of this note is to show that the order of the antipode of a Hopf algebra is not necessarily 2, but can be any positive even integer or infinite.

A coalgebra over a field $K$ is a vector space $C$ over $K$ together with maps $\delta: C \rightarrow C \otimes C$ and $\epsilon: C \rightarrow K$ satisfying

$$
(\delta \otimes 1) \delta=(1 \otimes \delta) \delta \text { and }(\epsilon \otimes 1) \delta=(1 \otimes \epsilon) \delta=1 .
$$

The coalgebra is cocommutative if $t \delta=\delta$, where $t: C \otimes C \rightarrow C \otimes C$ is defined by $t(c \otimes d)=d \otimes c$. A Hopf algebra over $K$ is an associative algebra $H$ with identity, together with identity-preserving algebra morphisms $\delta: H \rightarrow H \otimes H$ and $\epsilon: H \rightarrow K$ which give the underlying vector space a coalgebra structure. If $H$ is an associative algebra, we will denote by $\mu: H \otimes H \rightarrow H$ the map defined by $\mu(h \otimes k)=h k$, and by $\eta: K \rightarrow H$ the map defined by $\eta(a)=a 1$. An antipode for the Hopf algebra $H$ is a map $\gamma: H \rightarrow H$ satisfying

$$
\mu(\gamma \otimes 1) \delta=\mu(1 \otimes \gamma) \delta=\eta \epsilon .
$$

If there exists an antipode $\gamma$ for the Hopf algebra $H$, then it is unique, and is a Hopf algebra antiendomorphism. If $H$ is either commutative or cocommutative, then $\gamma^{2}=1$. (See $\$ 8$ of [1] for proofs of these facts.) The,$r d e r$ of the antipode $\gamma$ is the smallest positive integer $n$ such that $\gamma^{n}=1$, if such an integer exists, and is infinite otherwise. Since $\gamma$ is an antiendomorphism, if $\gamma$ has finite order it must have even order, unless $H$ is both commutative and cocommutative, in which case $\boldsymbol{\gamma}$ may have order 1 .

TheOREM. If $n$ is a positive even integer or infinite, there exists a Hopf algebra over $R$ which has an antipode of order $n$.

Free Hopf algebras over coalgebras. If $X$ is a vector space over $K$, we denote by $T(X)$ the tensor algebra of $X$. Let $C$ with the maps $\delta, \epsilon$ be a coalgebra. The map $C \rightarrow T(C) \otimes T(C)$ given by $c \rightarrow \delta(c)$ (where we are identifying $C \otimes C$ with a subspace of $T(C) \otimes T(C)$ by means of the usual identification of $C$ with a subspace of $T(C)$ ) induces an algebra morphism $\delta_{T}: T(C) \rightarrow T(C) \otimes T(C)$. Also the map $\epsilon: C \rightarrow K$ induces an

Received by the editors March 12, 1968.

${ }^{1}$ This research was partially supported by National Science Foundation grant NSF GP 8390. 
algebra morphism $\epsilon_{T}: T(C) \rightarrow K$. It is easily seen that $T(C)$ together with the maps $\delta_{T}$ and $\epsilon_{T}$ is a Hopf algebra, called the free Hopf algebra on $C$.

An antiautomorphism of the coalgebra $C$ is a bijective map $\zeta: C \rightarrow C$ satisfying

$$
\delta \zeta=(\zeta \otimes \zeta) t \delta \text { and } \epsilon \zeta=\epsilon .
$$

Given an antiautomorphism of $C$, consider the ideal $I C T(C)$ generated by all elements of the form

$$
\epsilon_{T}(c) 1-\mu_{T}(\zeta \otimes 1) \delta_{T}(c) \text { and } \epsilon_{T}(c) 1-\mu_{T}(1 \otimes \zeta) \delta_{T}(c),
$$

where $c \in C \subset T(C)$. It is easily checked that $\delta(I) \subset T(C) \otimes I+I \otimes T(C)$ and that $\epsilon(I)=0$. This implies that $H(C ; \zeta)=T(C) / I$ together with the maps $\delta_{H}, \epsilon_{H}$ induced by $\delta_{T}, \epsilon_{T}$ is a Hopf algebra. If $\kappa$ is the unique antiendomorphism of $T(C)$ satisfying $\kappa \mid C=\zeta$, then $\kappa(I) C I$, so $\kappa$ induces a map $\gamma_{H}: H(C ; \zeta) \rightarrow H(C ; \zeta)$.

The facts that $\gamma_{H}$ is an algebra antiendomorphism and that

$$
\mu_{H}\left(\gamma_{H} \otimes 1\right) \delta_{H}(c+I)=\mu_{H}\left(1 \otimes \gamma_{H}\right) \delta_{H}(c+I)=\eta_{H} \epsilon_{H}(c+I)
$$

for all $c \in C$ imply that $\gamma_{H}$ is an antipode for the Hopf algebra $H(C ; \zeta)$. The Hopf algebra $H(C ; \zeta)$ is called the free Hopf algebra with antipode on $C$ and $\zeta$. Denote by $\pi: C \rightarrow H(C ; \zeta)$ the composition of the maps $C \rightarrow T(C) \rightarrow H(C ; \zeta)$.

Warning. The map $\pi: C \rightarrow H(C ; \zeta)$ need not be injective.

The following Proposition is immediate:

PRoposition. Let $C$ be a coalgebra, and let $\zeta$ be an antiautomorphism of $C$. Then there exist a Hopf algebra $H(C ; \zeta)$ with antipode $\gamma$, and a morphism of coalgebras $\pi: C \rightarrow H(C ; \zeta)$ with $\pi \zeta=\gamma \pi$, such that for every Hopf algebra $H$ with antipode $\gamma^{\prime}$ and every coalgebra morphism $f: C \rightarrow H$ satisfying $\gamma^{\prime} f=f \zeta$, there exists a unique Hopf algebra morphism $g: H(C ; \zeta) \rightarrow H$ with $g \pi=f$.

Construction of the example. We prove the Theorem as follows: we construct a coalgebra $C$ with antiautomorphism $\zeta$ of order $n$ such that $\pi: C \rightarrow H(C ; \zeta)$ is injective. Then $\gamma\left|\pi(C)=\pi \zeta \pi^{-1}\right| \pi(C)$ is of order $n$, so that $\gamma$ is of order at least $n$. On the other hand, it is clear that $\gamma$ is of order at most $n$.

To construct $C$ and $\zeta$ we construct a finite dimensional algebra $A$ over $R$ with an antiautomorphism $\sigma$ of order $n$, and define $C=A^{*}$ $=\operatorname{hom}(A, R), \delta=\mu^{t}, \epsilon=\eta^{t}$, and $\zeta=\sigma^{t}$. If $A$ has a basis $\left\{a_{i}\right\}$ and a multiplication table $a_{i} a_{j}=\sum m_{i j k} a_{k}$, then $\delta$ is given explicitly by $\delta\left(a_{k}^{*}\right)=\sum m_{i j k} a_{i}^{*} \otimes a_{j}^{*}$, where $\left\{a_{i}^{*}\right\}$ is the basis of $C$ dual to the basis 
$\left\{a_{i}\right\}$. If $1=\sum e_{i} a_{i}$, then $\epsilon\left(a_{i}^{*}\right)=e_{i}$. If $\sigma\left(a_{i}\right)=\sum s_{i j} a_{j}$, then $\zeta\left(a_{j}^{*}\right)$ $=\sum s_{i j} a_{i}^{*}$. Thus, in this case $H(C ; \zeta)$ can be described as the associative algebra generated by $\left\{a_{i}^{*}\right\}$, subject to the relations

$$
e_{i}=\sum m_{l k i} s_{j l} a_{j}^{* *} a_{k}^{*} \text { and } e_{i}=\sum m_{j l i} s_{k l} a_{j}^{* *} a_{k}^{*} \text {. }
$$

To prove that $\pi: C \rightarrow H(C ; \zeta)$ is injective, it is sufficient to find a representation $\rho: H(C, \zeta) \rightarrow \operatorname{hom}(V, V)$ such that $\left\{\rho\left(a_{i}^{*}\right)\right\}$ is linearly independent.

Let $A$ be the algebra of all $2 \times 2$ matrices over $R$. If $n$ is a positive even integer, let $\theta=2 \pi / n$. If $n$ is infinite, let $\theta=\alpha \pi$, where $\alpha$ is any irrational number. Let

$$
U=\left|\begin{array}{cc}
\cos (\theta / 2) & \sin (\theta / 2) \\
-\sin (\theta / 2) & \cos (\theta / 2)
\end{array}\right|
$$

Define $\sigma: A \rightarrow A$ by $\sigma(T)=U^{-1} T^{\imath} U$, for all $T \in A$. The matrices

$$
\begin{aligned}
C^{\prime} & =\left|\begin{array}{ll}
1 & 0 \\
0 & 1
\end{array}\right|, \quad S^{\prime}=\left|\begin{array}{rr}
0 & 1 \\
-1 & 0
\end{array}\right|, \\
X^{\prime} & =\left|\begin{array}{ll}
0 & 1 \\
1 & 0
\end{array}\right|, \quad Y^{\prime}=\left|\begin{array}{rr}
-1 & 0 \\
0 & 1
\end{array}\right|,
\end{aligned}
$$

are a basis for $A$. With respect to this basis, $\sigma\left(C^{\prime}\right)=C^{\prime}, \sigma\left(S^{\prime}\right)=-S^{\prime}$, $\sigma\left(X^{\prime}\right)=\cos \theta X^{\prime}+\sin \theta Y^{\prime}$, and $\sigma\left(Y^{\prime}\right)=-\sin \theta X^{\prime}+\cos \theta Y^{\prime}$. It is clear that the antiautomorphism $\sigma$ is of order $n$.

Let $C, S, X$, and $Y$ be the basis of $C=A^{*}$ dual to the given basis of $A$. We apply the discussion in the second paragraph of this section to show that $H(C ; \zeta)$ is the algebra generated by $C, S, X$, and $Y$, subject to the relations

(1) $1=C^{2}+S^{2}+X \gamma(X)+Y \gamma(Y)$,

(2) $1=C^{2}+S^{2}+\gamma(X) X+\gamma(Y) Y$,

(3) $0=-C S+S C+X \gamma(Y)-Y \gamma(X)$,

(4) $0=C S-S C+\gamma(X) Y-\gamma(Y) X$,

(5) $0=C \gamma(X)+S \gamma(Y)+X C+Y S$,

(6) $0=C X-S Y+\gamma(X) C-\gamma(Y) S$,

(7) $0=C \gamma(Y)-S \gamma(X)-X S+Y C$,

(8) $0=C Y+S X+\gamma(X) S+\gamma(Y) C$, where $\gamma(X)=\cos \theta X-\sin \theta Y$ and $\gamma(Y)=\sin \theta X+\cos \theta Y$.

Some straightforward calculations show that equations (1)-(4) are equivalent to

(9) $X Y=Y X$,

(10) $1=C^{2}+S^{2}+\cos \theta\left(X^{2}+Y^{2}\right)$,

(11) $0=C S-S C-\sin \theta\left(X^{2}+Y^{2}\right)$, 
and that the equations (5)-(8) are equivalent to

(12) $0=C X+\cos \theta X C-\sin \theta X S$,

(13) $0=S X+\sin \theta X C+\cos \theta X S$,

(14) $0=C Y+\cos \theta Y C-\sin \theta Y S$,

(15) $0=S Y+\sin \theta Y C+\cos \theta Y S$.

Therefore $H(C ; \zeta)$ is the algebra generated by $C, S, X$, and $Y$, subject to the slightly less formidable relations (9)-(15).

We now define the representation $\rho$. Consider the representation of $T(C)$ on a three dimensional vector space defined by

$$
\begin{array}{clc}
C \rightarrow\left|\begin{array}{ccc}
1 & 0 & 0 \\
0 & -\cos \theta & 0 \\
0 & 0 & 1
\end{array}\right|, & S \rightarrow\left|\begin{array}{ccc}
0 & 0 & 0 \\
0 & -\sin \theta & 0 \\
0 & 0 & 0
\end{array}\right|, \\
X \rightarrow\left|\begin{array}{lll}
0 & 0 & 0 \\
1 & 0 & 0 \\
0 & 0 & 0
\end{array}\right|, & Y \rightarrow\left|\begin{array}{lll}
0 & 0 & 0 \\
0 & 0 & 1 \\
0 & 0 & 0
\end{array}\right| \vdots
\end{array}
$$

Routine calculations show that this representation preserves the relations $(9)-(15)$ and so induces a representation $\rho$ of $H(C ; \zeta)$ on this vector space. Clearly $\rho(C), \rho(S), \rho(X)$ and $\rho(Y)$ are linearly independent if $n>2$. Therefore the antipode of $H(C ; \zeta)$ has order $n$. Q.E.D.

\section{REFERENCE}

1. J. W. Milnor and J. C. Moore, On the structure of Hopf algebras, Ann. of Math. 81 (1965), 211-264.

University of Illinois at Chicago Circle 